\title{
Comparison of RT-PPP and RT-PPP-AR Methods
}

\author{
${ }^{1}$ Salih Alcay, ${ }^{* 1}$ Omer Faruk Atiz and ${ }^{1}$ Sermet Ogutcu, \\ ${ }^{* 1}$ Engineering and Architecture Faculty, Geomatics Engineering Department Necmettin Erbakan University, Turkey
}

\begin{abstract}
The Precise Point Positioning (PPP) is an absolute positioning method which emerged as an alternative to the relative positioning method. This method is widely used in many applications in recent years. In this method the static and kinematic positioning can be performed with high accuracy by using single GNSS receiver. In the last few years, the PPP method has started to be used in real time (RT-PPP) thanks to the real-time precise products (satellite orbit and clock data) produced by different organizations like IGS, BKG, CNES, ESA, EUREF, GFZ etc. The high interest in RT-PPP has accelerated the development of this method and many software have been developed. Recently, some of the software have ability to apply integer ambiguity resolution (AR). For this purpose, in this study the positioning performance of RT-PPP method was tested using two options of PPP-WIZARD software (RT-PPP, RT-PPP-AR). Three IGS stations were chosen in different geographical regions. The real-time coordinates of the stations belong to the observation period of about 4 hours were derived simultaneously with the RT-PPP and RT-PPP-AR options in 1 second epoch interval. The results obtained are given in a comparative approach.
\end{abstract}

Key words: IGS, RT-PPP, RT-PPP-AR, PPP-WIZARD

\section{Introduction}

In general, two types of positioning methods are used with GNSS, relative and absolute. Relative positioning methods are used in many engineering applications that require high accuracy. The traditional Real Time Kinematic method, which is one of the relative positioning methods, and the Network-RTK method developed afterwards are widely used in real-time applications [1, 2]. In the traditional RTK method, an accuracy in cm-level can be obtained by using a reference and rovers. The base length is limited to about $10 \mathrm{~km}$ when correction information is performed via radio connection, and when provided via the Internet, there is no distance restriction. In the NetworkRTK method, the reference receiver is not needed, and the corrections calculated from the network are transmitted to the receiver via the internet (GSM) connection. Also in this method, real-time position information is transmitted to the user with an accuracy of $\mathrm{cm}$ level.

In recent years, the interest in PPP method, which is one of the absolute positioning methods, has increased. In this method, position can be determined by using a single GNSS receiver. Many studies have been carried out on the performance of the Post Process PPP method [3, 4, 5, 6, 7, 8]. With static and kinematic PPP, a position accuracy of $\mathrm{cm}-\mathrm{dm}$ level can be achieved. In recent years, PPP method has started to be used in real time with the IGS-RT service offering orbit and clock products instantly $[9,10,11,12]$. In this study, in order to examine whether the RT-PPP-AR option has superiority comparing to the RT-PPP, both options of PPP-WIZARD software were tested.

*Corresponding author: Address: Engineering and Architecture Faculty, Geomatics Engineering Department Necmettin Erbakan University, Turkey 42140, Konya TURKEY. E-mail address: oatiz@erbakan.edu.tr 


\section{Real-Time Software Packages}

With the increasing interest in RT-PPP method, various software packages have been developed. The mostly used software packages are given below. In recent years, AR option can be used some of the software packages. Among them, PPP-WIZARD and PANDA can realize integer ambiguity solution.

- PPP-WIZARD (Precise Point Positioning with Integer and Zero-difference Ambiguity Resolution Demonstrator) [13]

- BKG Ntrip Client (BNC) (v2.12.6) [14]

- RTNET (Real Time NETwork processing Engine) [15]

- P3 [16]

- G-NUT/TEFNUT [17]

- PANDA (Positioning and Navigation Data Analyst) [18]

- RTKLIB [19]

Since PPP-WIZARD software is used in this study, details of this software and the options used are given in the subsection.

\subsection{PPP-WIZARD}

PPP-WIZARD software was developed by National Center for Space Studies (CNES). The software can perform integer ambiguity resolution (AR). The full AR can only be performed using GPS observations. The software has gap-bridging feature, and thanks to this feature, data gaps are removed [20]. In addition, the software can simultaneously process the data of three receivers. More details of the software can be found on the CNES website [13]. Details of the options used in the application are provided in Table 1.

Table 1. The options used in the processes

\begin{tabular}{cc}
\hline Parameter & PPP-WIZARD v1.4.2 \\
\hline Method & RT-PPP / RT-PPP-AR \\
Satellite system & $G P S$ \\
Cutoff & $10^{\circ}$ \\
Sampling interval & 1 second \\
Integer ambiguity resolution & With PPP-AR option \\
Zenith hydrostatic delay (ZHD) & Saastamoinen \\
Mapping Function (MF) & 1/sin(e); : Elevation angle \\
Orbit/Clock correction & SSRAOOCNEO \\
Orbit/Clock correction format & RTCM-SSR \\
Broadcast ephemeris & BCEPOOBKGO \\
Antenna phase center correction & igs 14. atx \\
Atmospheric tide loading & No \\
Solid earth tide & Yes \\
Phase wind-up & Yes \\
\hline
\end{tabular}




\section{Results and Analysis}

In this study, three IGS RT stations (PICL, KOUG, HOB2) in different parts of the world were used. The location of the stations are given in Fig. 1.

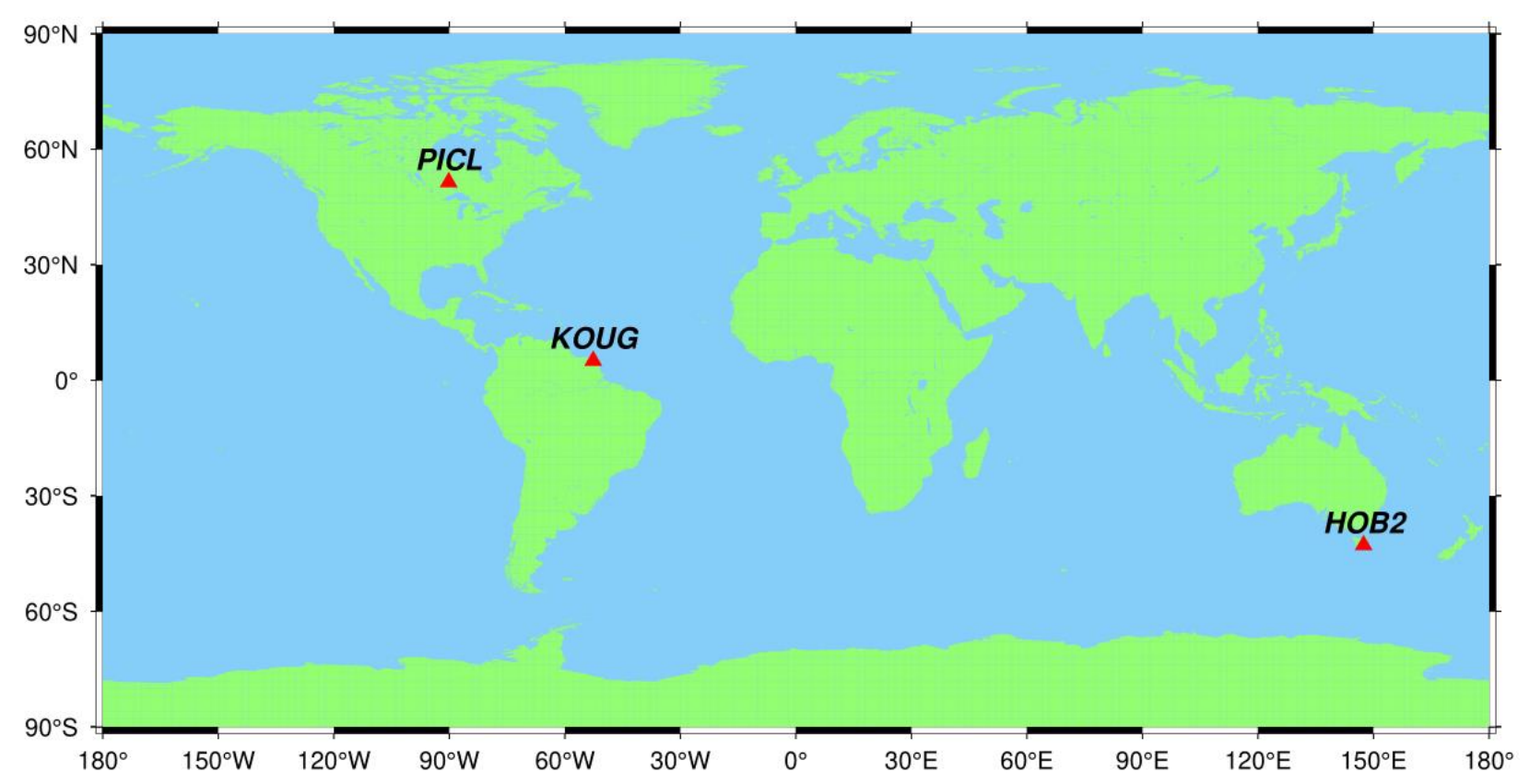

Figure 1. Location of the IGS stations

The processes were carried out for approximately 4 hours on 17 June 2020 using two options of PPP-WIZARD software (RT-PPP and RT-PPP-AR). In order to determine whether there was any ionosphere-induced storm during the application, geomagnetic storm (kp) [21], geomagnetic activity (dst) [22] and solar activity (F10.7) [23] indices were examined and it was determined that there was no storm condition. RT coordinate values of the stations were obtained in ITRF 2014 reference system in 1 second intervals. Since the PPP-WIZARD software can perform the full ambiguity resolution only using GPS, only GPS data were used in the processes. Then, measurement epoch coordinates of the stations were obtained from the IGS analysis center. These coordinates were taken as reference coordinates and real time coordinates obtained in each epoch were examined according to these coordinates. For this purpose, using the reference coordinates and real-time coordinates of the stations, a transformation was made from the cartesian coordinate system to the topocentric coordinate system, which is expressed north (n), east (e) and up (u) components. These components also represent the difference of RT-PPP coordinates from the reference coordinates.

Topocentric coordinates of the stations according to the two options (RT-PPP and RT-PPP-AR) are given in Fig. 2-4. 

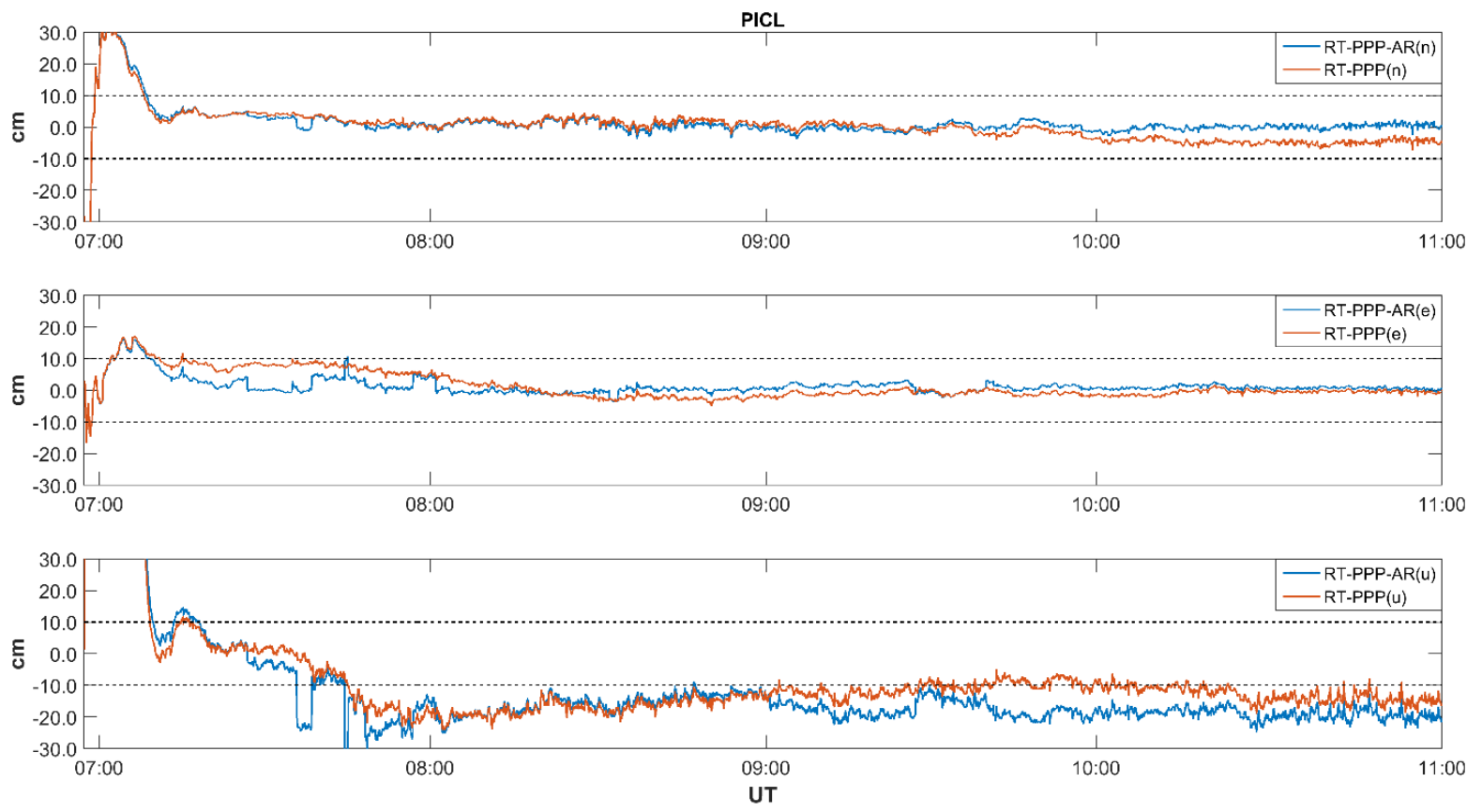

Figure 2. RT-PPP and RT-PPP-AR results of PICL station

In Fig. 2, coordinates obtained from RT-PPP and RT-PPP-AR methods belonging to the PICL station are given. It is seen that the accuracy of $\pm 10 \mathrm{~cm}$ are achieved after a convergence time of approximately 20 minutes by two methods in all components. However after about 07:45 UT the accuracy of up (u) component reach above $10 \mathrm{~cm}$ for both options. While RT-PPP-AR results are better than RT-PPP in horizontal components, RT-PPP provides better accuracy after 09:00 UT in vertical component.
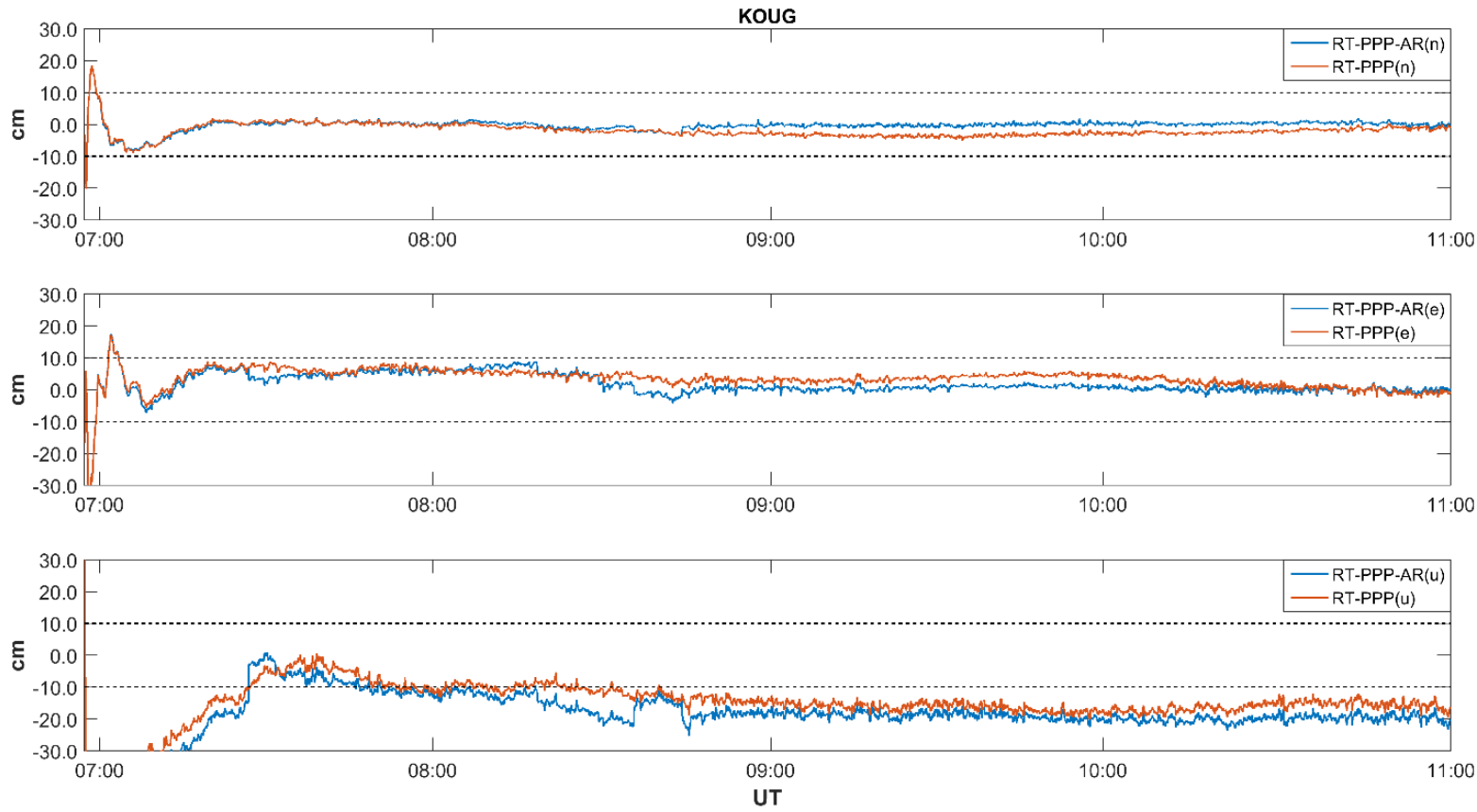

Figure 3. RT-PPP and RT-PPP-AR results of KOUG station 
The results corresponding to KOUG station are given in Fig. 3. It is seen that the convergence time in horizontal components ( $\sim 10 \mathrm{~min})$ appears to be shorter than the vertical component $(\sim 25 \mathrm{~min})$. After the convergence times, better accuracy of $10 \mathrm{~cm}$ and $20 \mathrm{~cm}$ is obtained in horizontal and vertical components, respectively, using both options. The differences between the RT-PPP-AR and RT-PPP results of this station are smaller than the PICL and HOB2.
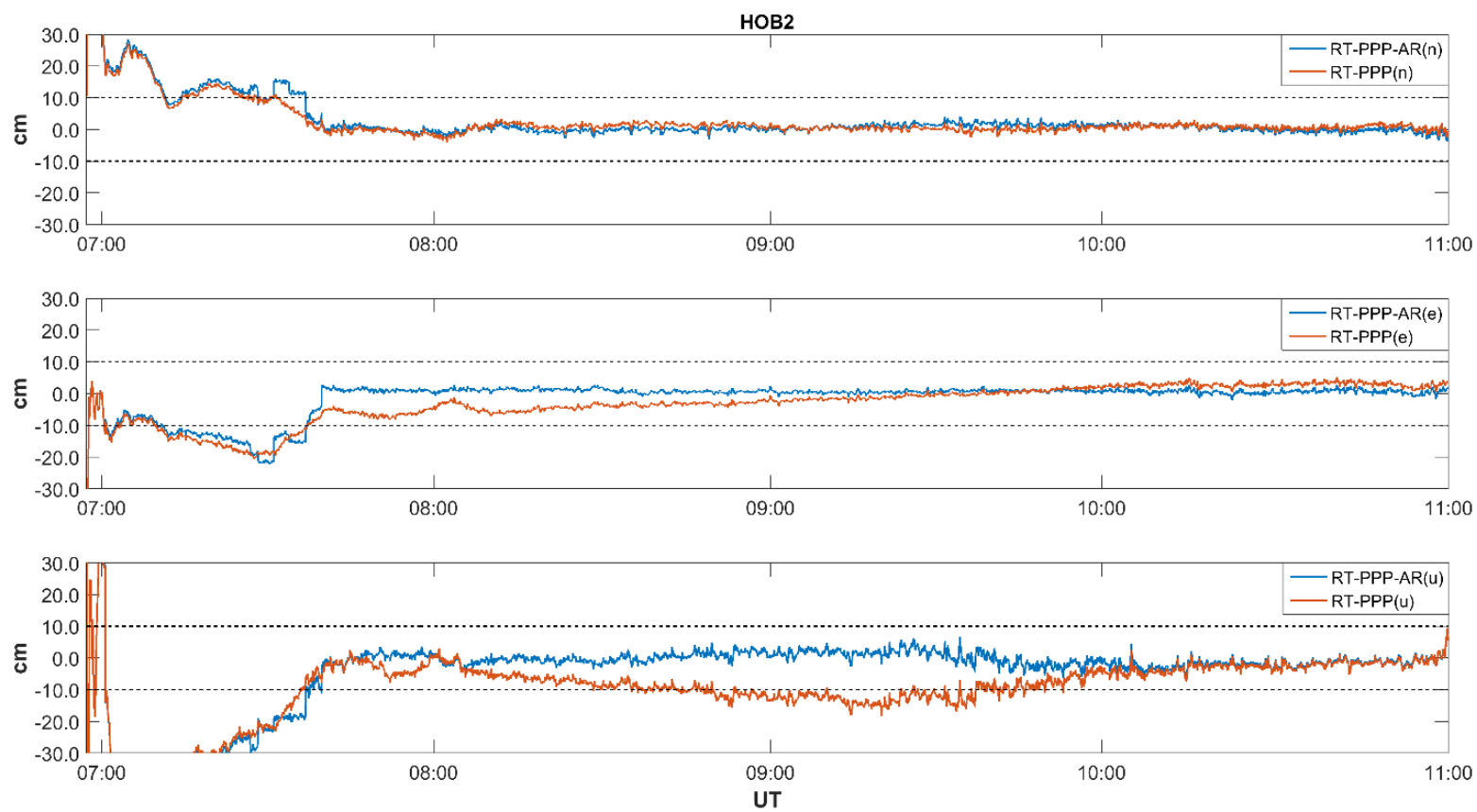

Figure 4. RT-PPP and RT-PPP-AR results of HOB2 station

When the coordinate values of HOB2 are examined, it is observed that convergence time is longer ( $\sim 35 \mathrm{~min}$ ) than PICL and KOUG. However, unlike other stations, the superiority of RT-PPP-AR is observed in vertical component, which provide $\pm 5 \mathrm{~cm}$ accuracy after convergence time. In addition, RT-PPP-AR results are better after 07:40 UT in all components and provides $\pm 2 \mathrm{~cm}$ accuracy in horizontal components.

In addition to examining the coordinates for accuracy, standard deviation (std) values for each coordinate components were calculated to test their repeatability. In addition to std, absolute maximum, absolute minimum and mean values were also calculated and given in Table 2. In calculation of mean and std values, first 30 minute, which includes convergence times, were not taken into consideration. 
Table 2. Basic statistical values of the coordinates

\begin{tabular}{llrrrrrr}
\hline \multirow{2}{*}{ Stations } & \multirow{2}{*}{ Basic Statistics } & \multicolumn{3}{c}{ RT-PPP-AR } & \multicolumn{3}{c}{ RT-PPP } \\
\cline { 3 - 8 } & & $\mathbf{n}(\mathbf{c m})$ & $\mathbf{e}(\mathbf{c m})$ & $\mathbf{u}(\mathbf{c m})$ & $\mathbf{n}(\mathbf{c m})$ & $\mathbf{e}(\mathbf{c m})$ & $\mathbf{u}(\mathbf{c m})$ \\
\hline \multirow{4}{*}{ PICL } & Maximum (abs) & 72.14 & 16.67 & 512.71 & 72.10 & 17.13 & 512.21 \\
& Minimum (abs) & 0.01 & 0.02 & 0.06 & 0.01 & 0.02 & 0.01 \\
& Mean & 0.38 & 0.73 & -17.17 & -0.73 & 0.36 & -13.03 \\
& Std & 1.28 & 1.40 & 4.23 & 2.95 & 3.08 & 4.58 \\
\hline \multirow{4}{*}{ KOUG } & Maximum (abs) & 20.19 & 42.32 & 234.11 & 20.19 & 42.32 & 234.78 \\
& Minimum (abs) & 0.01 & 0.01 & 0.07 & 0.01 & 0.01 & 0.01 \\
& Mean & -0.07 & 1.78 & -16.82 & -1.97 & 3.58 & -13.44 \\
& Std & 0.80 & 2.62 & 4.39 & 1.42 & 2.15 & 4.04 \\
\hline \multirow{4}{*}{ HOB2 } & Maximum (abs) & 95.22 & -69.96 & -78.69 & 95.22 & 69.96 & 80.41 \\
& Minimum (abs) & 0.01 & 0.01 & 0.01 & 0.01 & 0.01 & 0.01 \\
& Mean & 0.88 & 0.12 & -1.20 & 0.82 & -1.59 & -6.90 \\
& Std & 2.49 & 3.23 & 4.09 & 1.67 & 4.26 & 4.82 \\
\hline
\end{tabular}

As seen in Table 2, similar results were obtained with RT-PPP-AR and RT-PPP in terms of absolute maximum and absolute minimum. When the mean values are analyzed, it is seen that the differences between RT-PPP-AR and RT-PPP are less than $2 \mathrm{~cm}$ in horizontal components and 6 $\mathrm{cm}$ in vertical component. The std values indicate that the repeatability of the horizontal coordinates is better than the vertical coordinates for both options.

\section{Conclusions}

This study examines the performance of RT-PPP and RT-PPP-AR methods using three IGS RTS stations, which provide RT data. PPP-WIZARD v1.4.2 was used for estimating the coordinates. The RT-PPP and RT-PPP-AR results were examined in terms of both accuracy and precision. The results obtained after convergence times exhibit sub-decimeter accuracy using both options in horizontal components. Although RT-PPP-AR method is expected to give better results in all components, the superiority of the options varies according to the stations and horizontal-vertical components. In addition, the std values that indicate the precision of the results are less than $5 \mathrm{~cm}$ for both options after the convergence time.

\section{Acknowledgements}

We would like to thank to National Center for Space Studies (CNES) for the used PPP-WIZARD software. We thank the IGS-RT service for the products used in the processes. 


\section{References}

[1] Martin, A., and McGovern, E. (2012). An evaluation of the performance of network RTK GNSS services in Ireland. International Federation of Surveyors (FIG) Working week 6th-10th May 2012.

[2] Zhang, K., Wu, F., Wu, S., Rizos, C., Roberts, C., Ge, L., and Ramm, P. (2006). Sparse or dense: Challenges of Australian network RTK. Proceedings of IGNSS 2006.

[3] Alcay, S., Ogutcu, S., Kalayci, I., Yigit, C.O. (2019). Displacement monitoring performance of relative positioning and Precise Point Positioning (PPP) methods using simulation apparatus. Advances in Space Research 63 (5), 1697-1707, https://doi.org/10.1016/j.asr.2018.11.003.

[4] Alkan, R.M., Saka, M.H., Ozulu, I.M., Ilci, V. (2017). Kinematic precise point positioning using GPS and GLONASS measurements in marine environments, Measurement, 109, 36-43, https://doi.org/10.1016/j.measurement.2017.05.054.

[5] Choy, S., Zhang, S., Lahaye, F., Héroux, P. (2013). A Comparison between GPS-only and combined GPS+GLONASS Precise Point Positioning. Journal of Spatial Science, 58 (2), 169-190, https://doi.org/10.1080/14498596.2013.808164.

[6] Kiliszek, D and Kroszczyn'ski, K. (2020). Performance of the precise point positioning method along with the development of GPS, GLONASS and Galileo systems, Measurement, 164, 108009, https://doi.org/10.1016/j.measurement.2020.108009.

[7] Ogutcu, S. (2020). Assessing the contribution of Galileo to GPS+GLONASS PPP: Towards full operational capability, Measurement, $151, \quad 107143$, https://doi.org/10.1016/j.measurement.2019.107143.

[8] Yigit, C.O, Gikas, V., Alcay, S., Ceylan, A. (2014). Performance evaluation of short to long term GPS, GLONASS and GPS/GLONASS post-processed PPP, Survey Review, 46 (3), 155-166, https://doi.org/10.1179/1752270613Y.0000000068.

[9] Ahmed, F., Va'clavovic, P., Teferle, F.N., Dous`a, J., Bingley, R., Laurichesse, D. (2016). Comparative analysis of real-time Precise Point Positioning zenith total delay estimates, GPS Solutions, 20, 187-199, https://doi.org/10.1007/s10291-014-0427-z.

[10] Krzan, G., and Przestrzelski, P. (2016). GPS/GLONASS Precise Point Positioning with IGS Real-time service product. Acta Geodynamica et Geomaterialia, 13 (1), 69-81 https://doi.org/10.13168/AGG.2015.0047.

[11] Alcay, S., and Turgut, M. (2017). Performance evaluation of real-time precise point positioning method. IOP Conf. Series: Earth and Environmental Science, 95, 032023, https://iopscience.iop.org/article/10.1088/1755-1315/95/3/032023. 
[12] Nie, Z., Liu, F., Gao, Y. (2020). Real-time precise point positioning with a low-cost dual -frequency GNSS device, GPS Solutions, 24, 9, https://doi.org/10.1007/s10291-019-0922-3.

[13] http://www.ppp-wizard.net/ Accessed: 14.07.2020

[14] https://igs.bkg.bund.de/ntrip/download Accessed: 14.07.2020

[15] https://www.gps-solutions.com/rtnet_software Accessed: 14.07.2020

[16] http://people.ucalgary.ca/ ygao/p3.htm Accessed: 14.07.2020

[17] http://www.pecny.cz/gop/index.php/gnss/sw/tefnut Accessed: 14.07 .2020

[18] http://gpscenter.whu.edu.cn/ Accessed: 14.07.2020

[19] http://www.rtklib.com/ Accessed: 14.07.2020

[20] Laurichesse, D., and Privat, A. (2015). An open-source PPP client implementation for the CNES PPP-WIZARD demonstrator, Proceedings of the ION GNSS+ 2015, September, Tampa, Florida.

[21] ftp://ftp.gfz-potsdam.de/pub/home/obs/kp-ap/tab/ Accessed: 14.07.2020

[22] http://wdc.kugi.kyoto-u.ac.jp/dstdir/index.html Accessed: 14.07.2020

[23] http://eng.sepc.ac.cn/F107Forecast.php Accessed: 14.07.2020 